\title{
Building a Semantic Transparency Dataset of Chinese Nominal Compounds: A Practice of Crowdsourcing Methodology
}

\author{
Shichang Wang, Chu-Ren Huang, Yao Yao, Angel Chan \\ Department of Chinese and Bilingual Studies \\ The Hong Kong Polytechnic University \\ Hung Hom, Kowloon, Hong Kong \\ shi-chang. wang@connect . polyu.hk \\ \{churen.huang, y.yao, angel.ws.chan\}@polyu.edu.hk
}

\begin{abstract}
This paper describes the work which aimed to create a semantic transparency dataset of Chinese nominal compounds (SemTransCNC 1.0) by crowdsourcing methodology. We firstly selected about 1,200 Chinese nominal compounds from a lexicon of modern Chinese and the Sinica Corpus. Then through a series of crowdsourcing experiments conducted on the Crowdflower platform, we successfully collected both overall semantic transparency and constituent semantic transparency data for each of them. According to our evaluation, the data quality is good. This work filled a gap in Chinese language resources and also practiced and explored the crowdsourcing methodology for linguistic experiment and language resource construction.
\end{abstract}

\section{Introduction}

The meaning of “马虎” (măhu, horse-tiger, “careless”) has nearly nothing to do with neither “马” (mă, 'horse') nor “虎” (hǔ, 'tiger'). However the meaning of “道路” (dàolù, road-way, 'road') is basically equal to “道” (dào, 'road') or “路” (lù, “way'). And there are intermediate cases too, for instance, “江 湖” (jiānghú, river-lake, 'all corners of the country'), its meaning is not equal to “江” (jiāng, 'river') plus “湖” (hú, 'lake'), but clear relatedness between them can be observed. This phenomenon is called semantic transparency of compounds. We distinguish between overall semantic transparency (OST) and constituent semantic transparency (CST). The semantic transparency of a compound, i.e., the overall semantic transparency, is the extent to which the compound retains its literal meaning in its actual meaning. The semantic transparency of a constituent of a compound, i.e., the constituent semantic transparency, is the extent to which the constituent retains its meaning in the actual meaning of the compound. Semantic similarity between the literal meaning and the actual meaning of a compound can be used to estimate the overall semantic transparency of a compound, for the more the literal meaning is retained in the actual meaning, the more similar they are. The same technique can be used to estimate constituent semantic transparency. Semantic transparency can be quantified; if we assign 0 to "fully opaque" and assign 1 to "fully transparent", then semantic transparency can be quantified as a closed interval $[0,1]$.

The quantitative analysis of semantic transparency must be supported by semantic transparency datasets. In previous semantic transparency related studies on Chinese compounds, some researchers created some datasets to support their own studies. But this kind of datasets are usually relatively small and restrictive, so cannot be used widely, for example, (徐彩华 and 李镗, 2001; Myers et al., 2004; 干 红梅, 2008; Mok, 2009), etc. Some datasets, although large enough and can be used in other studies, are not publicly accessible, for example, (王春茂 and 彭聑龄, 1999; 高兵 and 高峰强, 2005), etc. A large and publicly accessible semantic transparency dataset of Chinese compounds is still a gap in Chinese language resources.

Crowdsourcing, as an emerging method of data collection and resource construction (Snow et al., 2008; Callison-Burch and Dredze, 2010; Munro et al., 2010; Schnoebelen and Kuperman, 2010; Gurevych and Zesch, 2013; Wang et al., 2013) and an emerging method of behavioral experiment (Paolacci et al., 2010;

This work is licenced under a Creative Commons Attribution 4.0 International License. Page numbers and proceedings footer are added by the organizers. License details: http://creativecommons.org/licenses/by/4.0/ 
Berinsky et al., 2011; Mason and Suri, 2012; Rand, 2012; Crump et al., 2013), is attracting more and more attention from the field of language study and language computing. As a method of data collection and resource construction, it has the advantages of high speed and low cost, etc. It can use redundancy to filter out noise in order to improve data quality; if used properly, it can produce expert-level data. As a method of experiment, besides the above advantages, it also has the following ones, (1) it is easier to obtain large samples, because the amount of potential participants is huge; (2) the diversity of participants is good, because the participants are from different places and have different backgrounds; (3) crowdsourcing environments are usually anonymous, so it is easier to collect certain sensitive data.

\section{Method}

\subsection{Compound Selection}

We use the following criteria to select compounds, (1) they are disyllabic nominal compounds; (2) each of them has the structure NN, AN, or VN; (3) they are composed of free morphemes; (4) they have mid-range word frequencies; and (5) they are used in both Mainland China and Taiwan. And we select compounds according to the following procedure:

(1) Extract monosyllabic nouns, adjectives and verbs mainly according to "The Dictionary of Contemporary Chinese (the 6th edition)" (现代汉语词典, 第 6 版), and thus we get three sets, a) the set of monosyllabic nouns, $\mathrm{N}$; b) the set of monosyllabic adjectives, A; and c) the set of monosyllabic verbs, $\mathrm{V}$.

(2) Extract the words of the structure NN, AN, or VN ${ }^{1}$ from the "Lexicon of Common Words in Contemporary Chinese" (现代汉语常用词表). In this step, NN means both morphemes of the word appear in the set N; AN means the first morpheme appears in the set A and the second appears in the set $\mathrm{N}$; VN means the first morpheme appears in the set $\mathrm{V}$ and the second appears in the set $\mathrm{N}$. After this step, we get "word list 1".

(3) Extract the words which have mid-range frequencies ${ }^{2}$ from the Sinica Corpus 4.0 (Chen et al., 1996). These words are represented in traditional Chinese characters. We convert them into simplified Chinese characters and only reserve the words which also appear in "word list 1". After this step, we get "word list 2".

(4) Manually verify "word list 2" to generate the final list. Things need to be verified include the following aspects. (a) Because in "word list 2" word structures are judged automatically, there are many errors, so we have to verify the correctness of the word structure judgments. (b) We have to make sure that the morphemes of each word are free morphemes. (c) We also need to delete some proper nouns.

The words we selected appear in both Sinica Corpus 4.0 and "Lexicon of Common Words in Contemporary Chinese". Since there is no completely reliable criterion to identify Chinese word, appearing in two lexicons ensures their word identity. This also ensures that they are used in both Mainland China and Taiwan, and further means they are quite possible to be shared in other Chinese language communities, for example Hong Kong, Macau, and Singapore, etc.

According to above criteria and procedure, we selected a total of 1,176 words. 664 (56.46\%) of them have the structure NN; 322 (27.38\%) have the structure AN; and $190(16.16 \%)$ have the structure VN.

\subsection{Experimental Design}

Normally, a crowdsourcing experiment should be reasonably small in size. We randomly divide these 1,176 words into 21 groups, $G_{i}(i=1,2,3, \ldots, 21)$; each group has 56 words.

\footnotetext{
${ }^{1}$ See 苑春法 and 黄昌宁 (1998), and Huang (1998) for relevant statistics.

${ }^{2}$ We use cumulative frequency feature to determine mid-range frequency. Sort the word frequency list of Sinica Corpus 4.0 descendingly; then calculate cumulative frequency word by word until each word corresponds with a cumulative frequency value; finally, plot a curve on a coordinate plane whose $\mathrm{x}$-axis represents the ranks of words in the sorted list, and the y-axis represents cumulative frequency values. Very apparently, this curve can be divided into three successive phases; the words within each phase have similar word frequency features. According to this, we identify three word frequency categories, 5,163 high-frequency words (frequency range: [182, 581823], cumulative frequency range: $[0 \%, 80 \%]), 19,803$ mid-range frequency words (frequency range: $[23,181]$, cumulative frequency range: $(80 \%, 93 \%])$, and 177,496 low-frequency words (frequency range: $[1,22]$, cumulative frequency range: $(93 \%, 100 \%])$. Sinica Corpus 4.0 contains about 11.2 million word tokens.
} 


\section{Questionnaires}

We collect overall semantic transparency (OST) and constituent semantic transparency (CST) data of these words. In order to avoid interaction, we designed two kinds of questionnaires to collect OST data and CST data respectively. So $G_{i}(i=1,2,3, \ldots, 21)$ has two questionnaires, one OST questionnaire for OST data collection and one CST questionnaire for CST data collection. Besides titles and instructions, each questionnaire has 3 sections. Section 1 is used to collect identity information includes gender, age, education and location. Section 2 contains four very simple questions about the Chinese language; the first two questions are open-ended Chinese character identification questions, the third question is a closeended homophonic character identification question, and the fourth one is a close-ended antonymous character identification question; different questionnaires use different questions. Section 3 contains the questions for semantic transparency data collection. Suppose $A B$ is a disyllabic nominal compound, we use the following question to collect its OST rating scores: "How is the sum of the meanings of $A$ and $B$ similar to the meaning of $A B$ ?" And use the following two questions to collect its CST rating scores of its two constituents: "How is the meaning of $A$ when it is used alone similar to its meaning in $A B$ ?" and "How is the meaning of $B$ when it is used alone similar to its meaning in $A B$ ?". 7-point scales are used in section 3; 1 means "not similar at all" and 7 means "almost the same".

In order to evaluate the data received in the experiments, we embedded some evaluation devices in the questionnaires. We mainly evaluated intra-group and inter-group consistency; and if the data have good intra-group and inter-group consistency, we can believe that the data quality is good. In each group we choose two words and make them appear twice, we call them intra-group repeated words and we can use them to evaluate the intra-group consistency. We insert into each group two same extra words, $w_{1}$ “地 步”, $w_{2}$ “高山”, to evaluate the inter-group consistency.

\section{Quality Control Measures}

On a crowdsourcing platform like Crowdflower, the participants are anonymous, they may try to cheat and submit invalid data, and they may come from different countries and speak different languages rather than the required one. There may be spammers who continuously submit invalid data at very high speed and they may even bypass the quality control measures to cheat for money. In order to ensure that the participants are native Chinese speakers and to improve data quality, we use the following measures, (1) a participant must correctly answer the first two Chinese character identification questions in the section $2 \mathrm{~s}$ of the questionnaires, and he/she must correctly answer at least one of the last two questions in these section $2 \mathrm{~s}$; (2) If a participant do not satisfy the above conditions, he/she will not see Section 3s; (3) each word stimulus in section $3 \mathrm{~s}$ has an option which allows the participants to skip it in case he/she does not recognize that word; (4) all the questions in the questionnaires must be answered except the ones which allow to be skipped and are explicitly claimed to be skipped; (5) we wrote a monitor program to detect and resist spammers automatically; (6) after the experiment is finished, we will analyze the data and filter out invalid data, and we will discuss this in detail in section 3 .

\subsection{Experimental Platform and Procedure}

We choose Crowdflower as our experimental platform, because according to our previous experiments, it is a feasible crowdsourcing platform to collect Chinese language data. We create one task for each questionnaire on the platform; there are 21 groups of word and each group has one OST questionnaire and one CST questionnaire, so there are a total of 42 tasks $T_{i}^{o s t}, T_{i}^{\text {cst }}(i=1,2,3, \ldots, 21)$. We publish these 42 tasks successively, and for each task we create a monitor program to detect and resist spammers. All of these tasks use the following parameters: (1) each task will collect 90 responses; (2) we pay 0.15 USD for each response of OST questionnaire and pay 0.25USD for each response of CST questionnaire; (3) each worker account of Crowdflower can only submit one response for each questionnaire and each IP address can only submit one response for each questionnaire; (4) we only allow the workers from the following regions (according to IP addresses) to submit data: Mainland China, Hong Kong, Macau, Taiwan, Singapore, Malaysia, USA, UK, Canada, Australia, Germany, France, Italy, New Zealand, and Indonesia; and we can dynamically disable or enable certain regions on demand in order to ensure both data quality and quantity. 


\section{Data Refinement and Result Calculation}

The OST dataset produced by the OST task $T_{i}^{o s t}(i=1,2,3, \ldots, 21)$ is $D_{i}^{\text {ost }}$. The CST dataset produced by the CST task $T_{i}^{\text {cst }}$ is $D_{i}^{\text {cst }}$. Each dataset contains 90 responses. Because of the nature of crowdsourcing environment, there are many invalid responses in each dataset; so firstly we need to filter them out in order to refine the data. A response is invalid if (1) its completion time is less than 135 seconds (for OST responses); its completion time is less than 250 seconds (for CST responses) ${ }^{3}$; or (2) it failed to correctly answer the first two questions of section $2 \mathrm{~s}$ of the questionnaires; or (3) it wrongly answered the last two questions of section $2 \mathrm{~s}$ of the questionnaires; or (4) it skipped one or more words in section $3 \mathrm{~s}$ of the questionnaires; or (5) it used less than two numbers on the 7-point scales in section $3 \mathrm{~s}$ of the questionnaires. The statistics of valid response are shown in Table 1.

The OST dataset $D_{i}^{\text {ost }}(i=1,2,3, \ldots, 21)$ contains $n_{i}$ valid responses; it means word $w$ in the OST dataset of the $i$ th group has $n_{i}$ OST rating scores; the arithmetic mean of these $n_{i}$ OST rating scores is the OST result of word $w$. The CST results of the two constituents of word $w$ are calculated using the same algorithm.

\begin{tabular}{cccccc}
\hline & \multicolumn{2}{c}{ OST } & & \multicolumn{2}{c}{ CST } \\
\cline { 2 - 3 } \cline { 5 - 6 }$G_{i}$ & $n$ & $\%$ & & $n$ & $\%$ \\
\hline$G_{1}$ & 54 & 60 & & 59 & 65.56 \\
$G_{2}$ & 60 & 66.67 & & 59 & 65.56 \\
$G_{3}$ & 55 & 61.11 & & 60 & 66.67 \\
$G_{4}$ & 59 & 65.56 & & 59 & 65.56 \\
$G_{5}$ & 50 & 55.56 & & 55 & 61.11 \\
$G_{6}$ & 55 & 61.11 & & 52 & 57.78 \\
$G_{7}$ & 53 & 58.89 & & 53 & 58.89 \\
$G_{8}$ & 60 & 66.67 & & 50 & 55.56 \\
$G_{9}$ & 48 & 53.33 & & 52 & 57.78 \\
$G_{10}$ & 57 & 63.33 & & 62 & 68.89 \\
$G_{11}$ & 46 & 51.11 & & 56 & 62.22 \\
$G_{12}$ & 48 & 53.33 & & 58 & 64.44 \\
$G_{13}$ & 51 & 56.67 & & 52 & 57.78 \\
$G_{14}$ & 50 & 55.56 & & 50 & 55.56 \\
$G_{15}$ & 52 & 57.78 & & 52 & 57.78 \\
$G_{16}$ & 57 & 63.33 & & 56 & 62.22 \\
$G_{17}$ & 50 & 55.56 & & 46 & 50.55 \\
$G_{18}$ & 51 & 56.67 & & 53 & 58.89 \\
$G_{19}$ & 50 & 55.56 & & 49 & 54.44 \\
$G_{20}$ & 50 & 55.56 & & 47 & 52.22 \\
$G_{21}$ & 50 & 55.56 & 50 & 55.56 \\
\hline Max & 60 & 66.67 & 62 & 68.89 \\
Min & 46 & 51.11 & & 46 & 50.55 \\
Median & 51.5 & 57.22 & 53 & 58.89 \\
Mean & 52.67 & 58.52 & & 53.81 & 59.76 \\
SD & 4.09 & 4.55 & & 4.49 & 5.04 \\
\hline
\end{tabular}

Table 1: The Amount of Valid Response in the OST and CST Datasets of Each Group

\section{Evaluation}

Three kinds of evaluation measures are used, (1) the intra-group consistency of the OST and CST results, (2) the inter-group consistency of the OST and CST results, and (3) the correlation between the OST and CST results.

\footnotetext{
${ }^{3}$ Each OST questionnaire has about 70 questions, and each CST questionnaire has about 130; in an OST or CST questionnaire, almost all the questions are the same except the stimuli words and can be instantly answered by intuition; note that a participant can take part in as many as 42 tasks; according to our test, if a participant is familiar with the tasks, he/she can answer each question in less than 2 seconds (less than 1 second to identify the stimulus word and another less than 1 second to rate it) without difficulty. $70 \times 2=140$ seconds, the expected time should be less than this, so we use 135 seconds as the temporal threshold for valid OST responses. The calculation of the temporal threshold for valid CST responses is similar, $130 \times 2=260$ seconds, the expected time should be less than this, so we use 250 seconds.
} 


\subsection{Intra-group Consistency}

In each group $G_{i}(i=1,2,3, \ldots, 21)$, we selected two words $w_{i, 1}, w_{i, 2}$ (intra-group repeated words) and made them appear twice between which there is enough distance; we can calculate the difference values between the results of the two appearances of these words.

\section{Intra-group Consistency of OST Results}

There are 21 groups and in each group there are two intra-group repeated words, so there are a total of 42 such words. Each intra-group repeated word appears twice, so we can obtain two OST results $r_{1}, r_{2}$. The difference value between the two results, $d=\left|r_{1}-r_{2}\right|$, of each intra-group repeated word is calculated, so there are 42 difference values. Among them, the maximum value is 0.29 ; the minimum value is 0 ; the median is 0.1 ; their mean is 0.11 ; and their standard deviation is 0.08 ; all of these values are low and indicate that these OST datasets have good intra-group consistency (see Table 2).

\section{Intra-group Consistency of CST Results}

Each intra-group repeated word has two constituents, $c_{1}, c_{2}$, so each constituent gets two CST results, i.e., $r_{c 1,1}, r_{c 1,2}$ and $r_{c 2,1}, r_{c 2,2}$. We calculate the difference values for the two constituents, $d_{1}=\left|r_{c 1,1}-r_{c 1,2}\right|$ and $d_{2}=\left|r_{c 2,1}-r_{c 2,2}\right|$, and get 42 difference values of the first constituents and 42 difference values of the second constituents. Among the difference values of the first constituents, the maximum value is 0.27 ; the minimum value is 0 ; the median is 0.09 ; their mean is 0.1 , and their standard deviation is 0.07; all of these values are low, this indicates that the CST results of the first constituents in the CST datasets of the 21 groups have good intra-group consistency. Among the difference values of the second constituents, the maximum value is 0.36 ; the minimum value is 0 ; the median is 0.07 ; their mean is 0.09 , and their standard deviation is 0.09 ; all of these values are low; this indicates that the CST results of the second constituents in the CST datasets of the 21 groups have good intra-group consistency (see Table 3). So these 21 CST datasets have good intra-group consistency.

\subsection{Inter-group Consistency}

We inserted two inter-group repeated words, $w_{1}$ “地步”, $w_{2}$ “高山”, into all of these 21 groups $G_{i}(i=$ $1,2,3, \ldots, 21)$; we can evaluate the inter-group consistency by comparing their semantic transparency rating results in different groups. Since $w_{1}, w_{2}$ appear in all OST and CST questionnaires of 21 groups, we can obtain (1) 21 OST results of $w_{1}$, (2) 21 OST results of $w_{2}$, (3) 21 CST results of each of the two constituents $w_{1, c 1}, w_{1, c 2}$ of $w_{1}$, and (4) 21 CST results of each of the two constituents $w_{2, c 1}, w_{2, c 2}$ of $w_{2}$. Standard deviation can be used to measure difference, for example, the standard deviation of the 21 OST results of $w_{1}$ is 0.2 ; this value is small and indicates high consistency; because these 21 results are from the OST datasets of 21 groups respectively, so we can say that these 21 OST datasets have good intergroup consistency. The standard deviation of the 21 OST results of $w_{2}$ is 0.14 ; the standard deviation of 21 CST results of the first constituent of $w_{1}$ is 0.2 , and that of the second is 0.18 ; the standard deviation of 21 CST results of the first constituent of $w_{2}$ is 0.15 , and that of the second is 0.2 ; all of these values are small and all of them indicate good inter-group consistency (see Table 4).

\subsection{Correlation between OST and CST Results}

Each compound in the datasets has two constituents; both constituents affect the OST of the compound, but neither of them can solely determine the OST of the compound. So the mean of the two CST values of a compound is a fairly good estimation of its OST value. Therefore, if the datasets are reliable, in each group, we should observe strong correlation between the OST results and their corresponding means of the CST results. For each group, we calculate three Pearson product-moment correlation coefficients $(r)$; $r_{1}$ is the $r$ between the OST results and their corresponding CST results of the first constituents; $r_{2}$ is the $r$ between the OST results and their corresponding CST results of the second constituents; and $r_{3}$ is the $r$ between the OST results and their corresponding means of the CST results. The $r_{3}$ values of the 21 groups are all greater than 0.9 which indicates very strong correlation; among them, the maximum value is 0.96 ; the minimum value is 0.91 ; and their mean is $0.94(S D=0.02)$; the $r_{1}$ and $r_{2}$ values are also 


\begin{tabular}{|c|c|c|c|c|}
\hline$G_{i}$ & $w_{i, 1 / 2}$ & $r_{1}$ & $r_{2}$ & $d$ \\
\hline \multirow{2}{*}{$G_{1}$} & 野狗 & 5.26 & 5.26 & 0 \\
\hline & 关节 & 3.57 & 3.61 & 0.04 \\
\hline \multirow{2}{*}{$G_{2}$} & 火灾 & 5.63 & 5.75 & 0.12 \\
\hline & 耳光 & 2.68 & 2.9 & 0.22 \\
\hline \multirow{2}{*}{$G_{3}$} & 笑脸 & 5.67 & 5.58 & 0.09 \\
\hline & 神气 & 3.51 & 3.62 & 0.11 \\
\hline \multirow{2}{*}{$G_{4}$} & 杂草 & 5.31 & 5.32 & 0.02 \\
\hline & 死党 & 3.19 & 3.02 & 0.17 \\
\hline \multirow{2}{*}{$G_{5}$} & 毒滤 & 5.36 & 5.32 & 0.04 \\
\hline & 水货 & 3.12 & 3.3 & 0.18 \\
\hline \multirow{2}{*}{$G_{6}$} & 手掌 & 5.53 & 5.4 & 0.13 \\
\hline & 火烧 & 5.25 & 4.96 & 0.29 \\
\hline \multirow{2}{*}{$G_{7}$} & 低价 & 5.25 & 5.23 & 0.02 \\
\hline & 黑洞 & 4.19 & 4.11 & 0.08 \\
\hline \multirow{2}{*}{$G_{8}$} & 凉风 & 5.48 & 5.33 & 0.15 \\
\hline & 风水 & 3.2 & 3.37 & 0.17 \\
\hline \multirow{2}{*}{$G_{9}$} & 琴声 & 5.19 & 5.19 & 0 \\
\hline & 手笔 & 3.69 & 3.75 & 0.06 \\
\hline \multirow{2}{*}{$G_{10}$} & 白云 & 5.49 & 5.63 & 0.14 \\
\hline & 风土 & 3.46 & 3.54 & 0.09 \\
\hline \multirow{2}{*}{$G_{11}$} & 雨伞 & 5.48 & 5.39 & 0.09 \\
\hline & 背心 & 3.26 & 3.24 & 0.02 \\
\hline \multirow{2}{*}{$G_{12}$} & 灯塔 & 5.19 & 5.4 & 0.21 \\
\hline & 脾气 & 3.6 & 3.54 & 0.06 \\
\hline \multirow{2}{*}{$G_{13}$} & 狂风 & 5.47 & 5.39 & 0.08 \\
\hline & 蓝本 & 3.37 & 3.41 & 0.04 \\
\hline \multirow{2}{*}{$G_{14}$} & 高楼 & 5.54 & 5.52 & 0.02 \\
\hline & 口角 & 3.46 & 3.56 & 0.1 \\
\hline \multirow{2}{*}{$G_{15}$} & 泥土 & 5.54 & 5.37 & 0.17 \\
\hline & 苦心 & 3.29 & 3.56 & 0.27 \\
\hline \multirow{2}{*}{$G_{16}$} & 鲜花 & 5.49 & 5.53 & 0.04 \\
\hline & 本分 & 3.82 & 4.07 & 0.25 \\
\hline \multirow{2}{*}{$G_{17}$} & 店主 & 5.2 & 5.38 & 0.18 \\
\hline & 香火 & 3.76 & 3.76 & 0 \\
\hline \multirow{2}{*}{$G_{18}$} & 桃花 & 5.31 & 5.18 & 0.14 \\
\hline & 色狼 & 3.41 & 3.25 & 0.16 \\
\hline \multirow{2}{*}{$G_{19}$} & 钱包 & 5.22 & 5.28 & 0.06 \\
\hline & 火气 & 4.04 & 3.88 & 0.16 \\
\hline \multirow{2}{*}{$G_{20}$} & 河岸 & 5.28 & 5.18 & 0.1 \\
\hline & 毛病 & 4.04 & 3.84 & 0.2 \\
\hline \multirow{7}{*}{$G_{21}$} & 古城 & 5.06 & 5.02 & 0.04 \\
\hline & 温床 & 3.8 & 4 & 0.2 \\
\hline & & & Max & 0.29 \\
\hline & & & Min & 0 \\
\hline & & & Median & 0.1 \\
\hline & & & Mean & 0.11 \\
\hline & & & SD & 0.08 \\
\hline
\end{tabular}

Table 2: The Intra-group Consistency of the OST Results of Each Group

reasonably high (see Table 5$)^{4}$. The results support the reliability of these datasets.

\section{$5 \quad$ Merging and Normalization}

The evaluation results show that the collected data are generally reliable and have relatively high intragroup and inter-group consistency which further indicate that these datasets share similar scale and are basically comparable, so we can merge the 21 OST datasets into one big OST dataset $D_{\text {ost }}$ and merge the 21 CST datasets into one big CST dataset $D_{c s t}$. When we merge these datasets, we delete all the extra words which are used to evaluate the inter-group consistency; for the repeated words which are

\footnotetext{
${ }^{4}$ After merging and normalization (see Section 5), we calculated these three correlation coefficients between $D_{\text {ost }}$ and $D_{c s t}$, the results are $r_{1}=0.68, r_{2}=0.68, r_{3}=0.87$.
} 


\begin{tabular}{|c|c|c|c|c|c|c|c|}
\hline \multirow[b]{2}{*}{$G_{i}$} & \multirow[b]{2}{*}{$w_{i, 1 / 2}$} & \multicolumn{3}{|c|}{$c_{1}$} & \multicolumn{3}{|c|}{$c_{2}$} \\
\hline & & $r_{c 1,1}$ & $r_{c 1,2}$ & $d_{1}$ & $r_{c 2,1}$ & $r_{c 2,2}$ & $d_{2}$ \\
\hline \multirow{2}{*}{$G_{1}$} & 野狗 & 3.83 & 4.05 & 0.22 & 5.49 & 5.42 & 0.07 \\
\hline & 关节 & 2.88 & 3.03 & 0.15 & 3.92 & 3.92 & 0 \\
\hline \multirow{2}{*}{$G_{2}$} & 火灾 & 5.12 & 5.22 & 0.1 & 5.24 & 5.1 & 0.14 \\
\hline & 耳光 & 4.27 & 4.27 & 0 & 2.19 & 2.51 & 0.32 \\
\hline \multirow{2}{*}{$G_{3}$} & 笑脸 & 5.12 & 5.08 & 0.03 & 5.35 & 5.4 & 0.05 \\
\hline & 神气 & 2.92 & 2.95 & 0.03 & 3.22 & 3.42 & 0.2 \\
\hline \multirow{2}{*}{$G_{4}$} & 杂草 & 4.51 & 4.34 & 0.17 & 5.56 & 5.27 & 0.29 \\
\hline & 死党 & 2.39 & 2.49 & 0.1 & 4.22 & 4.12 & 0.1 \\
\hline \multirow{2}{*}{$G_{5}$} & 毒瘾 & 4.75 & 4.64 & 0.11 & 5.09 & 5.15 & 0.05 \\
\hline & 水货 & 2.29 & 2.4 & 0.11 & 4.67 & 4.76 & 0.09 \\
\hline \multirow{2}{*}{$G_{6}$} & 手掌 & 5.4 & 5.23 & 0.17 & 5.35 & 5.4 & 0.06 \\
\hline & 火烧 & 5.08 & 5.02 & 0.06 & 5.38 & 5.46 & 0.08 \\
\hline \multirow{2}{*}{$G_{7}$} & 低价 & 4.7 & 4.83 & 0.13 & 5.13 & 5.13 & 0 \\
\hline & 黑洞 & 3.85 & 3.94 & 0.09 & 4.45 & 4.57 & 0.11 \\
\hline \multirow{2}{*}{$G_{8}$} & 凉风 & 5.06 & 4.88 & 0.18 & 5.28 & 5.3 & 0.02 \\
\hline & 风水 & 3.24 & 3.14 & 0.1 & 3.36 & 3.16 & 0.2 \\
\hline \multirow{2}{*}{$G_{9}$} & 琴声 & 5 & 4.98 & 0.02 & 5 & 4.98 & 0.02 \\
\hline & 手笔 & 3.63 & 3.71 & 0.08 & 3.71 & 3.83 & 0.12 \\
\hline \multirow{2}{*}{$G_{10}$} & 白云 & 4.53 & 4.6 & 0.06 & 5.37 & 5.39 & 0.02 \\
\hline & 风土 & 3.13 & 3.21 & 0.08 & 3.15 & 3.16 & 0.02 \\
\hline \multirow{2}{*}{$G_{11}$} & 雨伞 & 4.45 & 4.55 & 0.11 & 5.36 & 5.55 & 0.2 \\
\hline & 背心 & 3.8 & 3.79 & 0.02 & 2.64 & 3 & 0.36 \\
\hline \multirow{2}{*}{$G_{12}$} & 灯塔 & 4.69 & 4.52 & 0.17 & 4.97 & 4.9 & 0.07 \\
\hline & 脾气 & 3.03 & 3.21 & 0.17 & 3.28 & 3.4 & 0.12 \\
\hline \multirow{2}{*}{$G_{13}$} & 狂风 & 4.15 & 4.19 & 0.04 & 5.15 & 5.27 & 0.12 \\
\hline & 蓝本 & 2.52 & 2.79 & 0.27 & 3.44 & 3.42 & 0.02 \\
\hline \multirow{2}{*}{$G_{14}$} & 高楼 & 4.42 & 4.36 & 0.06 & 5.14 & 5.12 & 0.02 \\
\hline & 口角 & 3.56 & 3.5 & 0.06 & 3.08 & 3.06 & 0.02 \\
\hline \multirow{2}{*}{$G_{15}$} & 泥土 & 5.08 & 5.02 & 0.06 & 5.06 & 5.13 & 0.08 \\
\hline & 苦心 & 3.21 & 3 & 0.21 & 3.46 & 3.5 & 0.04 \\
\hline \multirow{2}{*}{$G_{16}$} & 鲜花 & 4.34 & 4.34 & 0 & 5.11 & 5.09 & 0.02 \\
\hline & 本分 & 3.8 & 3.63 & 0.18 & 3.32 & 3.38 & 0.05 \\
\hline \multirow{2}{*}{$G_{17}$} & 店主 & 4.76 & 4.72 & 0.04 & 4.74 & 4.87 & 0.13 \\
\hline & 香火 & 3.93 & 3.96 & 0.02 & 3.89 & 3.87 & 0.02 \\
\hline & 桃花 & 4.26 & 4.32 & 0.06 & 4.77 & 4.7 & 0.08 \\
\hline$G_{18}$ & 色狼 & 3.4 & 3.36 & 0.04 & 2.74 & 2.68 & 0.06 \\
\hline & 钱包 & 4.63 & 4.61 & 0.02 & 4.57 & 4.49 & 0.08 \\
\hline$G_{19}$ & 火气 & 3.55 & 3.29 & 0.27 & 3.53 & 3.41 & 0.12 \\
\hline & 河岸 & 4.98 & 4.91 & 0.06 & 5.15 & 5.17 & 0.02 \\
\hline$G_{20}$ & 毛病 & 2.94 & 2.96 & 0.02 & 4.7 & 4.45 & 0.26 \\
\hline & 古城 & 4.68 & 4.56 & 0.12 & 5 & 4.98 & 0.02 \\
\hline$G_{21}$ & 温床 & 3.68 & 3.88 & 0.2 & 3.66 & 3.6 & 0.06 \\
\hline & & & Max & 0.27 & & & 0.36 \\
\hline & & & Min & 0 & & & 0 \\
\hline & & & Median & 0.09 & & & 0.07 \\
\hline & & & Mean & 0.1 & & & 0.09 \\
\hline & & & SD & 0.07 & & & 0.09 \\
\hline
\end{tabular}

Table 3: The Intra-group Consistency of the CST Results of Each Group

used to evaluate the intra-group consistency, the final result of each of them is the mean of its two results. According to our definition, the range of semantic transparency value is $[0,1]$, but the experimental results are obtained using 7-point scales, so we need to normalize these results in order to map them to the range $[0,1]$. The normalized OST and CST results will be merged into $D_{o s t}$ and $D_{c s t}$ respectively. Assume that, in the dataset $D_{o s t}$, the OST result of the $i$ th $(i=1,2,3, \ldots, 1176)$ word is $S_{i}^{w}$, and the normalized result is $S_{i}^{\prime w}$, then,

$$
S_{i}^{\prime w}=\frac{S_{i}^{w}-1}{6}
$$




\begin{tabular}{|c|c|c|c|c|c|c|}
\hline \multirow[b]{2}{*}{$G_{i}$} & \multicolumn{2}{|c|}{ OST } & \multicolumn{4}{|c|}{ CST } \\
\hline & $w_{1}$ & $w_{2}$ & $w_{1, c 1}$ & $w_{1, c 2}$ & $w_{2, c 1}$ & $w_{2, c 2}$ \\
\hline$G_{1}$ & 2.94 & 5.52 & 2.85 & 2.97 & 4.56 & 5.56 \\
\hline$G_{2}$ & 3.6 & 5.55 & 3.15 & 3.2 & 4.92 & 5.75 \\
\hline$G_{3}$ & 3.51 & 5.64 & 3.17 & 3.23 & 4.75 & 5.58 \\
\hline$G_{4}$ & 3.81 & 5.68 & 3.53 & 3.59 & 4.58 & 5.42 \\
\hline$G_{5}$ & 3.74 & 5.46 & 3.38 & 3.56 & 4.64 & 5.55 \\
\hline$G_{6}$ & 3.65 & 5.55 & 3.63 & 3.56 & 4.85 & 5.65 \\
\hline$G_{7}$ & 3.58 & 5.51 & 3.47 & 3.58 & 4.75 & 5.23 \\
\hline$G_{8}$ & 3.22 & 5.53 & 3.4 & 3.36 & 4.8 & 5.48 \\
\hline$G_{9}$ & 3.31 & 5.15 & 3.48 & 3.52 & 4.69 & 5.42 \\
\hline$G_{10}$ & 3.58 & 5.53 & 3.42 & 3.34 & 4.69 & 5.27 \\
\hline$G_{11}$ & 3.7 & 5.67 & 3.46 & 3.32 & 4.52 & 5.36 \\
\hline$G_{12}$ & 3.33 & 5.71 & 3.19 & 3.28 & 4.41 & 5.14 \\
\hline$G_{13}$ & 3.47 & 5.78 & 3.58 & 3.56 & 4.73 & 5.38 \\
\hline$G_{14}$ & 3.48 & 5.58 & 2.94 & 2.94 & 4.42 & 5.3 \\
\hline$G_{15}$ & 3.4 & 5.42 & 3.42 & 3.27 & 4.62 & 5.1 \\
\hline$G_{16}$ & 3.47 & 5.56 & 3.34 & 3.25 & 4.59 & 5.16 \\
\hline$G_{17}$ & 3.6 & 5.56 & 3.3 & 3.26 & 4.5 & 5.17 \\
\hline$G_{18}$ & 3.67 & 5.67 & 3.36 & 3.34 & 4.47 & 5 \\
\hline$G_{19}$ & 3.28 & 5.56 & 3.2 & 3.29 & 4.37 & 5.18 \\
\hline$G_{20}$ & 3.56 & 5.48 & 3.21 & 3.36 & 4.72 & 5.34 \\
\hline$G_{21}$ & 3.62 & 5.32 & 3.2 & 3.28 & 4.5 & 5.24 \\
\hline Max & 3.81 & 5.78 & 3.63 & 3.59 & 4.92 & 5.75 \\
\hline Min & 2.94 & 5.15 & 2.85 & 2.94 & 4.37 & 5 \\
\hline Median & 3.56 & 5.55 & 3.36 & 3.32 & 4.62 & 5.34 \\
\hline Mean & 3.5 & 5.54 & 3.32 & 3.34 & 4.62 & 5.35 \\
\hline SD & 0.2 & 0.14 & 0.2 & 0.18 & 0.15 & 0.2 \\
\hline
\end{tabular}

Table 4: The Inter-group Consistency of the OST and CST Results

And assume that, in the dataset $D_{c s t}$, the CST result of the $j$ th $(j=1,2)$ constituent of the $i$ th word is $S_{i, j}^{c}$, and the normalized result is $S_{i, j}^{c}$, then,

$$
S_{i, j}^{\prime c}=\frac{S_{i, j}^{c}-1}{6}
$$

\section{Distribution}

Influenced by outliers and perhaps other factors, the OST and CST results cannot cover the whole range of the scale $[0,1]$; both ends shrink towards the central point 0.5 , and the shrinkage of each end is about 0.2 ; nevertheless, the results can still assign proper ranks of semantic transparency to the compounds and their constituents which are generally consistent with our intuitions. Among the normalized OST results, the maximum is 0.81 ; the minimum is 0.28 ; the median is 0.63 ; and their mean is $0.62(S D=0.09)$. Among the normalized CST results of the first constituents (C1.CST results), the maximum is 0.77; the minimum is 0.19 ; the median is 0.57 ; and their mean is $0.56(S D=0.09)$. And among the normalized CST results of the second constituents (C2.CST results), the maximum is 0.79; the minimum is 0.22 ; the median is 0.6 ; and their mean is $0.58(S D=0.1)$. The distributions of OST, C1.CST, and C2.CST results are similar; all of them are negatively skewed (see Figure 1), and their estimated skewnesses are -0.66 , -0.77 , and -0.63 respectively. These distributions exhibit that more compounds and their constituents in our datasets have relatively high semantic transparency values.

\section{Conclusion}

This work created a dataset of semantic transparency of Chinese nominal compounds (SemTransCNC 1.0), which filled a gap in Chinese language resources. It contains the overall and constituent semantic transparency data of about 1,200 Chinese disyllabic nominal compounds and can support semantic transparency related studies of Chinese compounds, for example, theoretical, statistical, psycholinguistic, and 


\begin{tabular}{cccc}
\hline$G_{i}$ & $r_{1}$ & $r_{2}$ & $r_{3}$ \\
\hline$G_{1}$ & 0.68 & 0.68 & 0.91 \\
$G_{2}$ & 0.72 & 0.72 & 0.93 \\
$G_{3}$ & 0.76 & 0.78 & 0.96 \\
$G_{4}$ & 0.76 & 0.77 & 0.96 \\
$G_{5}$ & 0.75 & 0.56 & 0.95 \\
$G_{6}$ & 0.63 & 0.72 & 0.91 \\
$G_{7}$ & 0.83 & 0.78 & 0.94 \\
$G_{8}$ & 0.76 & 0.77 & 0.96 \\
$G_{9}$ & 0.68 & 0.81 & 0.95 \\
$G_{10}$ & 0.84 & 0.83 & 0.95 \\
$G_{11}$ & 0.78 & 0.71 & 0.91 \\
$G_{12}$ & 0.72 & 0.77 & 0.95 \\
$G_{13}$ & 0.85 & 0.86 & 0.96 \\
$G_{14}$ & 0.69 & 0.85 & 0.95 \\
$G_{15}$ & 0.68 & 0.82 & 0.95 \\
$G_{16}$ & 0.82 & 0.85 & 0.95 \\
$G_{17}$ & 0.79 & 0.83 & 0.94 \\
$G_{18}$ & 0.81 & 0.86 & 0.96 \\
$G_{19}$ & 0.76 & 0.8 & 0.95 \\
$G_{20}$ & 0.76 & 0.75 & 0.94 \\
$G_{21}$ & 0.73 & 0.86 & 0.96 \\
\hline Max & 0.85 & 0.86 & 0.96 \\
Min & 0.63 & 0.56 & 0.91 \\
Median & 0.76 & 0.78 & 0.95 \\
Mean & 0.75 & 0.78 & 0.94 \\
SD & 0.06 & 0.07 & 0.02 \\
\hline & & &
\end{tabular}

Table 5: The Correlation Coefficients between the OST and CST Results
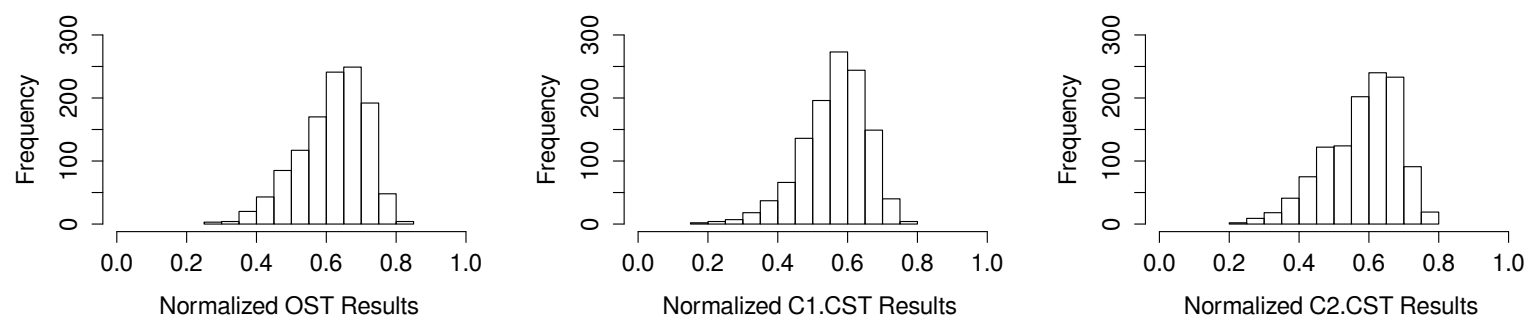

Figure 1: The Distributions of the Normalized OST and CST Results

computational studies, etc. And this work was also a successful practice of crowdsourcing method for linguistic experiment and language resource construction. Large scale language data collection experiments which require large amount of participants are usually very difficult to conduct in laboratories using the traditional paradigm. Crowdsourcing method enabled us to finish the data collection task within relatively short period of time and relatively low budget (1,000USD); during the process of the experiment, we needed not to organize and communicate with the participants, it saved a lot of time and energy. The participants are from all over the world, so it is better than traditional laboratory method in the aspect of participant diversity. The data collected have very good intra-group and inter-group consistency, the OST and CST data highly correlate with each other as expected, and the results are consistent with our intuitions: all of these indicate good data quality. The methods of questionnaire design, quality control, data refinement, evaluation, emerging, and normalization can be used in crowdsourcing practices of the same kind. 


\section{Acknowledgements}

The work described in this paper was supported by grants from the Research Grants Council of the Hong Kong Special Administrative Region, China (Project No. 544011 \& 543512).

\section{References}

Adam J Berinsky, Gregory A Huber, and Gabriel S Lenz. 2011. Using mechanical turk as a subject recruitment tool for experimental research. Submitted for review.

Chris Callison-Burch and Mark Dredze. 2010. Creating speech and language data with amazon's mechanical turk. In Proceedings of the NAACL HLT 2010 Workshop on Creating Speech and Language Data with Amazon's Mechanical Turk, pages 1-12. Association for Computational Linguistics.

Keh-Jiann Chen, Chu-Ren Huang, Li-Ping Chang, and Hui-Li Hsu. 1996. Sinica Corpus: Design Methodology for Balanced Corpora. In B.-S. Park and J.B. Kim, editors, Proceeding of the 11th Pacific Asia Conference on Language, Information and Computation, pages 167-176. Seoul:Kyung Hee University.

Matthew JC Crump, John V McDonnell, and Todd M Gureckis. 2013. Evaluating amazon's mechanical turk as a tool for experimental behavioral research. PloS one, 8(3):e57410.

Iryna Gurevych and Torsten Zesch. 2013. Collective intelligence and language resources: introduction to the special issue on collaboratively constructed language resources. Language Resources and Evaluation, 47(1):17.

Shuanfan Huang. 1998. Chinese as a headless language in compounding morphology. New approaches to Chinese word formation: Morphology, phonology and the lexicon in modern and ancient Chinese, pages 261-284.

Winter Mason and Siddharth Suri. 2012. Conducting behavioral research on amazon’ s mechanical turk. Behavior research methods, 44(1):1-23.

Leh Woon Mok. 2009. Word-superiority effect as a function of semantic transparency of chinese bimorphemic compound words. Language and Cognitive Processes, 24(7-8):1039-1081.

Robert Munro, Steven Bethard, Victor Kuperman, Vicky Tzuyin Lai, Robin Melnick, Christopher Potts, Tyler Schnoebelen, and Harry Tily. 2010. Crowdsourcing and language studies: the new generation of linguistic data. In Proceedings of the NAACL HLT 2010 Workshop on Creating Speech and Language Data with Amazon's Mechanical Turk, pages 122-130. Association for Computational Linguistics.

James Myers, Bruce Derwing, and Gary Libben. 2004. The effect of priming direction on reading chinese compounds. Mental Lexicon Working Papers, 1:69-86.

Gabriele Paolacci, Jesse Chandler, and Panagiotis G Ipeirotis. 2010. Running experiments on amazon mechanical turk. Judgment and Decision making, 5(5):411-419.

David G Rand. 2012. The promise of mechanical turk: How online labor markets can help theorists run behavioral experiments. Journal of theoretical biology, 299:172-179.

Tyler Schnoebelen and Victor Kuperman. 2010. Using amazon mechanical turk for linguistic research. Psihologija, 43(4):441-464.

Rion Snow, Brendan O'Connor, Daniel Jurafsky, and Andrew Y Ng. 2008. Cheap and fast—but is it good?: evaluating non-expert annotations for natural language tasks. In Proceedings of the conference on empirical methods in natural language processing, pages 254-263. Association for Computational Linguistics.

Aobo Wang, Cong Duy Vu Hoang, and Min-Yen Kan. 2013. Perspectives on crowdsourcing annotations for natural language processing. Language Resources and Evaluation, 47:9-31.

干红梅. 2008. 语义透明度对中级汉语阅读中词汇学习的影响. 语言文字应用, 1:82-90.

徐彩华 and 李镗. 2001. 语义透明度影响儿童词汇学习的实验研究. 语言文字应用, 1:53-59.

王春茂 and 彭聘龄. 1999. 合成词加工中的词频, 词素频率及语义透明度. 心理学报, 31(3):266-273.

苑春法 and 黄昌宁. 1998. 基于语素数据库的汉语语素及构词研究. 世界汉语教学, 2(1):13.

高兵 and 高峰强. 2005. 汉语字词识别中词频和语义透明度的交互作用. 心理科学, 28(6):1358-1360. 